\title{
ESCALAS CONCORRENTES PARA A MENSURACC̃̃O DA QUALIDADE PERCEBIDA: UMA COMPARAÇÃO ENTRE A SERVQUAL E A RSQ
}

COMPETING SCALES FOR MEASURING THE PERCEIVED QUALITY: A COMPARISON BETWEEN THE SERVQUAL AND RSQ

\section{RESUMO}

Não há dúvida sobre a importância da qualidade de serviços como fator de sucesso empresarial, mas mensurar essa qualidade tem se mostrado um desafio quando se consideram diferentes contextos. Diante disso, o objetivo deste estudo foi testar duas escalas de mensuração da qualidade percebida de serviços. A comparação entre a escala Service Quality (Servqual) e a Retail Service Quality (RSQ), deu-se por meio de survey junto a 351 respondentes e utilizou como ambiente de pesquisa uma rede de home centers com lojas localizadas na cidade de São Paulo. Para analisar os dados obtidos, foram utilizadas as técnicas multivariadas de análise fatorial exploratória e confirmatória. Como resultado, as duas escalas demonstraram níveis aceitáveis de confiabilidade e validade. Entretanto, no teste de validade nomológica, a escala RSQ mostrou-se superior à escala Servqual, uma vez que a primeira foi capaz de explicar 43\% da lealdade em relação ao varejista, enquanto a segunda explicou apenas $11 \%$.

\section{Evandro Luiz Lopes}

Doutorando pelo Programa de Mestrado e Doutorado, Universidade Nove de Julho - SP, Brasil

elldijo@uol.com.br

\section{José Mauro da Costa Hernandez}

Professor da Escola de Artes e Ciências Humanas, Universidade de São Paulo - SP, Brasil

jmh@uol.com.br

\section{Jouliana Jordan Nohara}

Professora do Programa de Mestrado e Doutorado, Universidade Nove de Julho - SP, Brasil

jnohara@uol.com.br

Recebido em 12.11.2008. Aprovado em 19.08.2009

Avaliado pelo sistema double blind review

Editor Científico: André Luiz Samartini

\begin{abstract}
There is no doubt about the importance of service quality as a factor of businesses' success, but to measure this quality has proved to be a challenge when one considers different environmental contexts. Given this, the main goal of this paper was to test two measurement scales of perceived service quality. The comparison between Service Quality scale (Servqual) and Retail Service Quality (RSQ) was conducted by means of a survey with 351 participants, clients of a home center stores chain located in the city of São Paulo. The data were analyzed using both exploratory and confirmatory factor analysis. As a result, both scales demonstrated acceptable levels of reliability and validity. However, the RSQ demonstrated a better performance in the nomological test since it was able to explain $43 \%$ of the loyalty towards the retailer, while the Servqual scale explained only $11 \%$.
\end{abstract}

PALAVRAS-CHAVE Qualidade percebida, Servqual, RSQ, escalas concorrentes, home centers

KEYWORDS Perceived quality, Servqual, RSQ, competing scales, home centers. 


\section{INTRODUÇÃO}

A importância do setor de serviços na estrutura econômica mundial tem alcançado patamares cada vez mais significativos (LOVELOCK e WRIGHT, 2001), resultando no aumento da competitividade entre as organizações e na necessidade de diferenciação entre os concorrentes (EPPLE e ZELENITZ, 1981).

Diversos autores entendem que a qualidade percebida por um consumidor pode ser um fator decisivo na escolha de um provedor de serviços (REIDENBACH e SANDIFERSMALLWOOD, 1990; CONGRAM e FRIEDMAN, 1991; GRÖNROOS, 1995). A qualidade percebida de serviços (QPS) geralmente é tida como um conceito puramente subjetivo, resultado de uma mescla de juízos, conhecimentos e expectativas (SHOSTACK, 1981; PARASURAMAN, ZEITHAML e BERRY, 1985; PARASURAMAN, ZEITHAML e BERRY, 1988). A QPS não está somente relacionada à satisfação do consumidor (REIDENBACH e SANDIFERSMALLWOOD, 1990), mas também é essencial para a consecução de dois objetivos principais de um negócio de serviços: atrair e reter os consumidores, disputandoos em um ambiente competitivo, e indicar aos gestores do negócio um mix de serviços otimizado, minimizando assim os custos operacionais.

Em relação ao primeiro objetivo - atração e retenção de clientes - entende-se que quando um serviço está disponível em fornecedores muito próximos e similares, como em uma concentração de lojas, por exemplo, a QPS assume o papel de fator diferenciador entre eles (CONGRAM e FRIEDMAN, 1991). Quanto ao segundo objetivo - otimização dos serviços oferecidos com a minimização dos custos operacionais - Grönroos (1995) defende que a qualidade não está diretamente relacionada ao aumento de custos, mas pode representar esse aumento caso não seja bem dimensionada ou executada.

Congram e Friedman (1991) sugerem que a qualidade está intimamente ligada à eficiência e à produtividade, e entregar serviços de forma eficiente evita a insatisfação dos consumidores, bem como os custos financeiros e humanos para reparar os danos causados pela prestação inadequada de um serviço. Daí a importância em se realizarem estudos visando entender a QPS, seus reflexos no comportamento dos consumidores e suas correlações com outros construtos latentes.

Por muitos anos, a principal escala de mensuração da qualidade de serviços foi a Servqual (PARASURAMAN, ZEITHAML e BERRY, 1988), que analisa a QPS por meio de três visões distintas: qualidade ideal, qualidade mínima exigida e qualidade observada de um determinado servi- ço. Mesmo sendo severamente criticada, quer seja pela crença de que a mensuração da qualidade percebida por si só já explicaria o desempenho do fornecedor de serviços e também a satisfação do consumidor, tornando desnecessária a mensuração da expectativa de desempenho (CRONIN e TAYLOR, 1992), quer seja pelo entendimento de que os atributos mensurados pela escala são do tipo vetorial, isto é, na expectativa do consumidor, o atributo em sua plenitude tenderia ao infinito (TEAS, 1994), a Servqual ainda é tida como a mais tradicional ferramenta para a mensuração da QPS (HUGHEY, CHAWLA e KHAN, 2003), já tendo sido replicada muitas vezes, em diversas ocasiões, no cenário varejista nacional e no internacional (por exemplo, NEL e PITT, 1993; MACHADO, QUEIROZ e MARTINS, 2006).

Mais recentemente, Dabholkar, Thorpe e Rentz (1996) propuseram uma escala de mensuração da percepção da qualidade de serviços aplicada ao varejo denominada Retail Service Quality - RSQ (Qualidade de Serviços no Varejo) por entenderem haver carência de um modelo específico para esse setor da economia. Desde então, vários pesquisadores têm utilizado a escala, buscando validar sua concepção teórica e sua aplicabilidade em formatos varejistas distintos. Todavia, não foram identificados trabalhos que tenham testado a escala RSQ no varejo brasileiro.

As críticas à escala Servqual e as diversas validações da escala RSQ, que pode constituir uma nova opção para a pesquisa da QPS no varejo, motivaram os autores deste estudo a desenvolverem uma comparação entre essas duas ferramentas, utilizando para isso um operador varejista brasileiro como cenário de pesquisa.

Para se atingir tal objetivo, estrutura-se o presente estudo em cinco seções. Na primeira seção são examinados os conceitos de qualidade de serviços e lealdade, além da contextualização do desenvolvimento das escalas propostas. Na seção a seguir explicita-se o método utilizado, enquanto na terceira seção se expõem os resultados da pesquisa de campo. Na quarta seção realiza-se a discussão dos resultados observados, e finalmente, na última seção apresentam-se as reflexões finais do trabalho.

\section{REFERENCIAL TEÓRICO}

Nesta seção serão abordados o conceito de qualidade percebida de serviços e os embasamentos teóricos e metodológicos das escalas Servqual (PARASURAMAN, ZEITHAML e BERRY, 1988) e RSQ (DABHOLKAR, THORPE E RENTZ, 1996). 


\section{Qualidade percebida de serviços}

A qualidade percebida de serviços (QPS) pode ser definida como a percepção dos consumidores acerca da qualidade ou superioridade de um produto ou serviço em relação à sua funcionalidade e às suas alternativas (LOURO, 2000). Galé (1996) define qualidade percebida como a opinião dos clientes sobre os produtos e serviços, comparativamente com os da concorrência. Segundo Dominguez (2000), a qualidade percebida pode ser entendida como o julgamento do consumidor sobre a excelência de um produto ou serviço. Ainda segundo esse autor, diferentemente do conceito de valor percebido, a qualidade percebida não envolve uma comparação entre os elementos dados (custos) e recebidos (benefícios).

Diversos pesquisadores consideram a qualidade de serviços como uma avaliação global, similar à atitude. Segundo eles, a semelhança parte do fato de as atitudes tratarem de um conceito global vinculado às predisposições individuais (PARASURAMAN; ZEITHAML; BERRY, 1988). Concordando com essa argumentação, Solomon (1998) define atitude como a predisposição em avaliar um objeto (ou produto) positiva ou negativamente.

Para Grönroos (1995), uma boa qualidade percebida é obtida quando a qualidade experimentada atende às expectativas do cliente, ou seja, a qualidade esperada. Portanto, o processo de avaliação da qualidade do serviço que o cliente faz é função de suas expectativas (qualidade esperada) e de sua percepção do serviço (qualidade experimentada). Parasuraman, Zeithaml e Berry (1988) afirmam que, na ausência de medidas objetivas, uma abordagem apropriada para mensurar a QPS oferecida pela empresa é medir a diferença entre as expectativas e a percepção dos consumidores acerca do desempenho da empresa.

\section{Escalas para a mensuração da qualidade percebida}

São apresentadas abaixo as duas escalas abordadas no presente trabalho.

\section{A escala Servqual}

Embasados nos estudos até então realizados, Parasuraman, Zeithaml e Berry (1988) desenvolveram uma escala denominada Service Quality Gap Analysis (Servqual), que leva em consideração as expectativas dos clientes em relação a um determinado serviço frente à percepção de qualidade do serviço recebido. A escala contém 22 pares de itens que podem ser agrupados em cinco dimensões da qualidade - aspectos tangíveis, confiabilidade, presteza, segurança e empatia. O primeiro item de cada par identifica o nível de desempenho esperado e o segundo identifica o nível de serviço percebido. A operacionalização da escala se dá pelo cálculo da diferença entre as percepções de desempenho e as expectativas de serviço. Para cada par de itens obtém-se um índice, que é definido como a diferença entre o serviço percebido e o serviço desejado, denominada por Parasuraman, Zeithaml e Berry (1994) Medida da Superioridade do Serviço (MSS). Esse índice pode ser positivo ou negativo, e pode-se afirmar que quanto maior for o índice, maior será a superioridade do serviço.

Dois aspectos tornam-se inegáveis sob a luz da literatura acadêmica. O primeiro diz respeito ao crescimento contínuo da utilização da metodologia proposta por Parasuraman, Zeithaml e Berry (1988), quer seja no campo acadêmico, quer seja no ambiente empresarial (PHILIP e HAZLETT, 1996; ESPINOZA, 1999). Urdan (1993) destaca a longevidade do modelo, alegando que o estudo realizado por Parasuraman, Zeithaml e Berry $(1985,1988)$ foi o mais sistemático e abrangente já concebido com o intuito de identificar as dimensões da qualidade de serviços, salientando também que outros modelos e escalas propostos parecem ter sido esquecidos pela literatura acadêmica, e que isso, no mínimo, indica uma superioridade da Servqual (URDAN, 1993). O segundo aspecto reside na sugestão existente em diversos trabalhos (CRONIN e TAYLOR, 1992; TEAS, 1994; FINN e LAMB, 1991) de novas pesquisas a fim de elucidar as dúvidas relacionadas à natureza dos atributos que compõem a escala Servqual.

Embora os criadores do instrumento afirmem que a escala Servqual é válida numa ampla variedade de empresas de serviço, quando aplicada a um único formato empresarial não poderá prescindir de adaptações em suas variáveis para ajustar-se à realidade do serviço estudado, ou seja, serão necessárias pequenas modificações nas 22 afirmativas, ajustando sua redação ao contexto específico (PARASURAMAN, ZEITHAML e BERRY, 1988).

\section{A escala RSQ}

Ao proporem uma nova escala para se mensurar a QPS em organizações varejistas, Dabholkar, Thorpe e Rentz (1996) entendiam que ainda não havia uma escala com a amplitude necessária que a levasse a ser credenciada para a mensuração da percepção de qualidade dos serviços em qualquer segmento de negócios. Segundo Dabholkar e seus colegas, o ambiente competitivo no varejo mudava rapidamente, pois eram crescentes tanto a intensificação da competição entre empresas domésticas e estrangeiras como o número de incorporações e aquisições.

Uma das opções, a escala Servqual, o instrumento mais intensamente conhecido para mensurar a QPS, havia sido testada empiricamente em empresas fornecedoras de serviços puros, tais como escolas e provedores de serviços 
bancários. Dessa forma, a escala se mostrava mais robusta apenas quando aplicada em empresas onde inexistia, ao menos em grande escala, a comercialização de produtos físicos. Ainda segundo Dabholkar e seus colegas, a Servqual não havia sido adaptada com sucesso na aplicação em um ambiente de loja de varejo, que possui características diferentes daquelas encontradas em empresas de serviços puros. Para eles, em empresas varejistas, os clientes percorrem a loja livremente fazendo seu próprio percurso, muitas vezes encontrando a mercadoria que querem de forma autônoma, interagindo com diversos funcionários da empresa, devolvendo e trocando mercadorias, entre outras características específicas que influenciam a avaliação da qualidade dos serviços sob a ótica dos clientes (DABHOLKAR, THORPE e RENTZ, 1996).

De fato, Dabholkar, Thorpe e Rentz (1996) notaram a pouca aderência da escala Servqual quando aplicada a ambientes varejistas (CARMAN, 1990; FINN e LAMB, 1991; GUIRY, HUTCHINSON e WEITZ, 1992) e por isso acreditaram que nesse ambiente se fazia necessário mensurar outras dimensões além daquelas apontadas na Servqual.

Julgando ser necessário conceber uma escala que possibilitasse a mensuração da QPS em empresas varejistas, Dabholkar, Thorpe e Rentz (1996) iniciaram uma triangulação de pesquisas empíricas. Na primeira pesquisa, por meio de entrevistas feitas com consumidores que haviam acabado de fazer uma compra, buscaram identificar os atributos da qualidade relativos à experiência de compra em lojas varejistas que eram significativos para os clientes. Esse estudo mostrou que os clientes estavam muito interessados na disposição física das lojas, pois eles próprios precisavam se movimentar pelos corredores e, muitas vezes, encontrar sozinhos os produtos desejados. Também havia grande interesse pelo tratamento recebido dos funcionários da loja, pelo conforto que ela proporcionava, pela política de aceitação de cartões de crédito e pela facilidade de troca ou devolução dos produtos comprados. Na segunda pesquisa, foram realizadas seis entrevistas em profundidade com frequentadores de lojas de varejo, visando descobrir outros atributos importantes que não haviam sido citados na primeira rodada de entrevistas. Vários atributos identificados na primeira pesquisa foram corroborados nessa rodada, entre eles a disposição dos corredores, o atendimento do pessoal da loja e a facilidade para devolver produtos. Entretanto, outros atributos também foram identificados: a aparência agradável da loja e das instalações (facilities, em inglês), tais como banheiros, provadores, estacionamentos, entre outros. Esses entrevistados também comentaram sobre a importância de que todas as promessas feitas pela loja fossem cumpridas.
Finalmente, a terceira pesquisa da triangulação qualitativa utilizou como técnica "seguir" os clientes dentro da loja e observar como eles se comportavam. Os entrevistados foram gravados enquanto caminhavam pelos corredores das lojas e interagiam com funcionários e com as mercadorias expostas. No momento em que decidiam adquirir algum produto, eles relatavam o que estavam pensando e o porquê de optarem por aquele produto e não por outro. Os autores alegam que a experiência de compra e as interações dos clientes com os demais elementos do ambiente foram observadas sem que houvesse alterações significativas no fluxo natural da compra. Os participantes comentavam sobre a aparência da loja, a disposição dos corredores, a disponibilidade e a qualidade do auxílio dos funcionários do varejista, e sobre a qualidade dos produtos ofertados.

Combinando as descobertas realizadas durante a etapa exploratória com a literatura sobre os pressupostos da escala Servqual, Dabholkar, Thorpe e Rentz (1996) conceberam uma estrutura hierárquica dos fatores que determinam a qualidade de serviço para o ambiente varejista. Da mesma forma que Parasuraman, Zeithaml e Berry (1985, 1988), também Dabholkar, Thorpe e Rentz (1996) sugerem que a QPS é determinada por cinco dimensões distintas. Entretanto, as dimensões preconizadas naquele estudo foram: aspectos físicos, confiabilidade, interações pessoais, solução de problemas e políticas internas do varejista. A escala proposta por Dabholkar, Thorpe e Rentz (1996) é composta por 28 questões, nas quais o respondente deve externar sua opinião relativa à qualidade percebida de um determinado serviço utilizando para tanto uma escala de Likert de 5 pontos.

Desde sua divulgação em 1996, a escala RSQ vem sendo utilizada como ferramenta para o aprofundamento do conhecimento sobre a QPS e sua correlação com outros construtos latentes, em operações varejistas ao redor do mundo (BLOSE, TANKERSLEY e FLYNN, 1999; METHA e outros, 1999; KIM e JIN, 2002; RAMAYAH e LEEN, 2003; KAUL, 2005).

Ainda que as escalas Servqual e RSQ convirjam para o entendimento de que a QPS é composta por cinco dimensões independentes, três diferenças conceituais podem ser verificadas: i) embora tanto a escala RSQ quanto a escala Servqual sejam compostas por cinco dimensões, elas diferenciam-se na concepção teórica e na quantidade de variáveis que as compõem (veja o Quadro 1); ii) enquanto a escala proposta por Parasuraman, Zeithaml e Berry (1988) analisa a QPS por meio da qualidade mínima aceitável, pela qualidade ideal e pela qualidade experimentada no serviço que está sendo analisado, a escala RSQ utiliza apenas a qualidade experimentada pelo usuário; iii) en- 
quanto para os criadores da Servqual o instrumento pode ser aplicado a qualquer tipo de serviço, para Dabholkar, Thorpe e Rentz a escala RSQ é indicada apenas para avaliação da QPS em ambientes varejistas tradicionais.

\section{MÉTODO}

Nesta seção são expostos todos os procedimentos metodológicos utilizados na etapa empírica deste trabalho.

\section{Escalas}

Para a mensuração da QPS foram utilizadas as escalas RSQ e Servqual. A escala RSQ utilizada foi traduzida do estudo realizado por Dabholkar, Thorpe e Rentz (1996). Já a escala Servqual (PARASURAMAN, ZEITHAML e BERRY, 1988) foi adaptada ao ambiente ao qual havia sido aplicada, como indicado pelos autores do instrumento.

Para testar a validade nomológica, ou seja, até que ponto construtos teoricamente distintos se relacionam de maneira empiricamente significativa (LEVIN e FOX, 2006), utilizou-se uma escala de lealdade construída a partir de três subescalas (intenção de reutilização, disposição do consumidor em recomendar positivamente o serviço e autodeclaração de lealdade), cujos itens foram adaptados de escalas já existentes (DICK e BASU, 1994; REICHHELD, 1996; MCMULLAN, 2005; SETH, MOMAYA e GUPTA, 2005; MAJUMDAR, 2005; REICHHELD, 2006). A intenção de recompra (de produtos) ou reutilização (de serviços) é um aspecto considerado como indissociável da existência de lealdade, pois se encontra no eixo central da própria definição que alguns pesquisadores fazem do construto (DICK e BASU, 1994). Dessa forma, o questionamento direto ao cliente sobre a intenção de recompra e reutilização apresenta-se como um indicador usual para a mensuração da lealdade. A predisposição para comunicação positiva por parte do consumidor no âmbito pessoal, denominada recomendação (REICHHELD, 1996; 2006), também é amplamente utilizada para a mensuração da lealdade, além da autodeclaração de lealdade ao revendedor (MCMULLAN, 2005; SETH, MOMAYA e GUPTA, 2005; MAJUMDAR, 2005). Os itens foram mensurados por uma escala do tipo Likert de 5 pontos $(1=$ discordo totalmente; 5 = concordo totalmente).

\section{Validação das escalas}

Como não foi encontrada nenhuma outra aplicação da escala RSQ no Brasil, utilizou-se o procedimento metodológico denominado validação de conteúdo (MALHOTRA, 2001). Após a tradução do inglês para o português, as 28 questões do modelo RSQ foram submetidas, em ordem aleatória, a sete doutores da área de Ciências Sociais aplicadas para que relacionassem cada afirmativa à sua respectiva dimensão, com o objetivo de assegurar a aderência do modelo ao cenário local. A validação de conteúdo foi satisfatória, visto que $92 \%$ das relações entre as 28 afirmativas e as dimensões foram apontadas corretamente, o que, em última análise, confirma a aplicabilidade da escala (MALHOTRA, 2001).

Como a metodologia proposta por Parasuraman, Zeithaml e Berry (1988) sugere a necessidade de adaptação do enunciado das 22 afirmativas quando é aplicada em um segmento específico, a versão adaptada da escala foi traduzida novamente para o inglês e submetida, por correio eletrônico, ao professor A. Parasuraman para que a validasse. A validação ocorreu em 26/05/2007 com a sugestão de que fossem mudadas as ancoragens da escala de Likert. Na versão enviada, a escala de Likert variava entre (1) concordo totalmente e (9) discordo totalmente. $\mathrm{Na}$ versão final, para melhor entendimento dos pesquisados e por sugestão de Parasuraman, os pontos extremos foram alterados para (1) baixa e (9) alta.

\section{Questionário}

Utilizou-se um formulário de pesquisa estruturado em torno de quatro seções. A primeira seção continha os 22 pares de questões para a mensuração da QPS sob a ótica da Servqual utilizando uma escala de Likert de 9 pontos. Na segunda parte do questionário, outra mensuração da QPS, porém utilizando-se os 28 itens da escala RSQ, mensurados por meio de uma escala de Likert de 5 pontos. A terceira seção continha os três itens para a mensuração da lealdade. Finalmente, as questões da quarta seção tinham o objetivo de identificar os dados sociodemográficos dos respondentes. O Quadro 1 detalha as variáveis utilizadas neste estudo.

\section{Amostra}

Foram entrevistados consumidores de materiais de construção civil de uma rede de home centers da cidade de São Paulo. Justifica-se a escolha de uma loja desse segmento por tratar-se de um dos mais representativos do setor varejista nacional (MORGADO e GONÇALVES, 1997). Além disso, as experiências de compra nas lojas dessa rede podem ser muito variadas, já que elas possuem tanto uma área de vendas dedicada ao autosserviço, o que possibilita ao consumidor adquirir produtos sem a necessidade de ajuda, quanto áreas de vendas assistidas nas quais a experiência de compra requer a atuação de um vendedor. As entrevistas foram feitas em 11 lojas para que os dados 


\section{ARTIGOS - ESCALAS CONCORRENTES PARA A MENSURAÇÃO DA QUALIDADE PERCEBIDA: UMA COMPARAÇÃO ENTRE A SERVQUAL E A RSQ}

Quadro 1 - Variáveis utilizadas

\begin{tabular}{|c|c|}
\hline & ESCALA SERVQUAL \\
\hline Tang1 & Equipamentos com aparência moderna \\
\hline Tang2 & Instalações físicas atraentes. \\
\hline Tang3 & Boa aparência dos funcionários \\
\hline Tang4 & Qualidade dos catálogos, manuais e a sinalização da loja. \\
\hline Conf5 & Manutenção dos serviços pelos prazos divulgados. \\
\hline Conf6 & Interesse em resolver os problemas dos clientes. \\
\hline Conf7 & $\begin{array}{l}\text { Fornecimento dos serviços combinados de maneira certa logo na } \\
\text { primeira vez. }\end{array}$ \\
\hline Conf8 & Cumprimento dos prazos combinados. \\
\hline Conf9 & $\begin{array}{l}\text { Manutenção e guarda dos registros comerciais e dos clientes sem } \\
\text { erros. }\end{array}$ \\
\hline Prest10 & $\begin{array}{l}\text { Informação correta de prazos de execução dos serviços pelos } \\
\text { funcionários }\end{array}$ \\
\hline Prest11 & Atendimento imediato dos clientes. \\
\hline Prest12 & Funcionários sempre dispostos a ajudar os clientes. \\
\hline Prest13 & Funcionários sempre livres para responder os pedidos dos clientes. \\
\hline Seg14 & Funcionários com postura que inspira confiança aos clientes. \\
\hline Seg15 & Funcionários bem treinados para atender os clientes. \\
\hline Seg16 & Funcionários cordiais com os clientes. \\
\hline Seg17 & $\begin{array}{l}\text { Funcionários com conhecimento para responder as dúvidas dos } \\
\text { clientes. }\end{array}$ \\
\hline Emp18 & Atenção individual aos clientes. \\
\hline Emp19 & Horário de funcionamento conveniente para os clientes. \\
\hline Emp20 & $\begin{array}{l}\text { Funcionários, na quantidade e com a qualidade necessária para dar } \\
\text { atenção pessoal aos clientes. }\end{array}$ \\
\hline Emp21 & Tratamento dos interesses do cliente como prioridade. \\
\hline Emp22 & Entendimento das necessidades específicas de seus clientes. \\
\hline
\end{tabular}

\section{ESCALA LEALDADE}

Leal1 Continuarei comprando nesta loja

Leal2 Recomendaria esta loja a parentes e amigos

Leal3 Considero-me leal a esta loja

\begin{tabular}{|c|c|}
\hline & ESCALA RSQ \\
\hline P1Afis & P1- Esta loja tem equipamentos e mobiliário modernos \\
\hline P2Afis & P2- Os ambientes de apoio da loja são visualmente agradáveis \\
\hline P3Afis & $\begin{array}{l}\text { P3- Os materiais relacionados a serviços (sacolas, catálogos, impressos) } \\
\text { são visualmente agradáveis }\end{array}$ \\
\hline P4Afis & $\begin{array}{l}\text { P4- A limpeza da loja, incluindo banheiros e salas de descanso, é } \\
\text { adequada }\end{array}$ \\
\hline P5Afis & P5- 0 layout da loja facilita o cliente encontrar o que necessita \\
\hline P6Afis & P6- 0 layout da loja facilita a locomoção do cliente \\
\hline P7Conf & P7- Quando a loja promete algo, cumpre dentro do prazo informado \\
\hline P8Conf & P8- A loja cumpre suas promessas. \\
\hline P9Conf & P9- A loja realiza os serviços de maneira correta na primeira vez \\
\hline P10Conf & P10- A loja mantém disponível as mercadorias que os clientes desejam \\
\hline P11Conf & $\begin{array}{l}\text { P11- A loja esforça-se para manter os registros de transações e dados } \\
\text { sem erros }\end{array}$ \\
\hline P12Pess & $\begin{array}{l}\text { P12- Os empregados desta loja têm condições de sanar as dúvidas } \\
\text { dos clientes }\end{array}$ \\
\hline P13Pess & P13- 0 comportamento dos funcionários inspira confiança aos clientes \\
\hline P14Pess & $\begin{array}{l}\text { P14- } 0 \text { cliente se sente seguro em realizar transações comerciais com } \\
\text { esta loja }\end{array}$ \\
\hline P15Pess & $\begin{array}{l}\text { P15- Os empregados desta loja estão sempre disponiveis a atender os } \\
\text { clientes }\end{array}$ \\
\hline P16Pess & $\begin{array}{l}\text { P16- Os empregados desta loja prestam informações corretas sobre os } \\
\text { prazos em que os serviços serão realizados }\end{array}$ \\
\hline P17Pess & $\begin{array}{l}\text { P17- Os empregados desta loja sempre estão disponíveis para sanar as } \\
\text { dúvidas dos clientes }\end{array}$ \\
\hline P18Pess & P18- Esta loja dá atendimento personalizado aos seus clientes \\
\hline P19Pess & P19- Os empregados desta loja sempre são cordiais \\
\hline P20Pess & P20- Os empregados desta loja sempre são cordiais ao telefone \\
\hline P21SProb & P21- A loja facilita a troca e devolução das mercadorias \\
\hline P22SProb & $\begin{array}{l}\text { P22- Quando o cliente tem um problema, a loja mostra interesse sin- } \\
\text { cero em resolver }\end{array}$ \\
\hline P23SProb & $\begin{array}{l}\text { P23- Os empregados da loja são habilitados a solucionar pessoalmente } \\
\text { as dúvidas e reclamações }\end{array}$ \\
\hline P24Pol & P24- Esta loja oferece produtos de alta qualidade \\
\hline P25Pol & P25- Esta loja disponibiliza amplo estacionamento \\
\hline P26Pol & $\begin{array}{l}\text { P26- } 0 \text { horário de funcionamento desta loja é conveniente para seus } \\
\text { clientes }\end{array}$ \\
\hline P27Pol & P27- A loja aceita a maioria dos cartões de crédito \\
\hline P28Pol & P28- A loja oferece seus próprios cartões de crédito \\
\hline
\end{tabular}


obtidos representassem da melhor forma possível a amplitude dos diferentes perfis de consumidores da rede.

\section{Procedimentos}

A coleta dos dados dos pesquisados ocorreu por meio do autopreenchimento do formulário, na saída das lojas, logo após a finalização das compras, como sugerido por Rust e Oliver (1994). Para mitigar a possibilidade de vieses, além da técnica de amostragem intencional por tráfego, na qual são selecionados os respondentes que, na opinião do pesquisador, possuem, a princípio, as características específicas que ele deseja ver refletidas em sua amostra (LAKATOS e MARCONI, 1996), foi adotado o procedimento de selecionar os elementos da amostra definindo-se a cota máxima de 10 respondentes por dia em cada ponto de venda, durante o horário de funcionamento das lojas, descartando-se pessoas com idade inferior a 18 anos.

Os clientes foram abordados quando finalizavam suas compras e convidados a participar da pesquisa em uma ambiente anexo ao salão de vendas, espaço utilizado para a realização de cursos e palestras que existe em todas as lojas do varejista. Os respondentes, após o preenchimento dos formulários, foram convidados a desfrutar de um lanche ofertado pela gerência de cada loja. Com o objetivo de estimular os clientes a participar da pesquisa, foi sorteado entre os respondentes um aparelho de DVD doado pela empresa.

\section{Análise dos dados}

Para elaborar a análise dos dados realizou-se a análise fatorial exploratória (AFE), com a utilização do SPSS 13.0, e a análise fatorial confirmatória (AFC), utilizando-se o Lisrel 8.51.

A AFE deu-se pelo método de análise de componentes principais e rotação oblíqua Direct Oblimin (com D = 0) e foi motivada por razões teóricas, visto que a natureza das dimensões pressupõe intercorrelações positivas e diferentes de zero. Nesta fase também foi feita a análise de confiabilidade de consistência interna por meio do cálculo do coeficiente alfa de Cronbach.

Os seguintes critérios foram adotados para a análise fatorial exploratória: comunalidade extraída dos itens superior a 0,4, teste de esfericidade de Bartlett significativo ao nível de 5\% e teste Kaiser-Meyer-Olkin (KMO) superior a 0,7 (HAIR e outros, 2005). Para o coeficiente alfa de Cronbach o valor mínimo aceitável foi 0,7 (NUNNALLY, 1978).

A AFC seguiu a metodologia proposta por Anderson e Gerbing (1988), sendo realizada em duas etapas. Na primeira, foram analisados os modelos de mensuração do conjunto de variáveis, buscando-se identificar sua validade convergente - o quanto os itens de um construto latente explicam sua variância total, expressa pelas matrizes lambda (l) superiores a 0,60 - e sua validade discriminante - ou seja, o quanto os construtos latentes são independentes entre si, expressa por valores da matriz phi (f) inferiores a 0,60 (ANDERSON e GERBING, 1988; KLINE, 2005). Por essa metodologia, os modelos são considerados apropriados quando, além das matrizes phi e lambda adequadas, apresentarem os índices de ajustamento CFI, GFI, NFI superiores a 0,90 e o RMR padronizado menor que 0,05 (KLINE, 2005), e ainda quando o RMSEA apre-

\section{Tabela 1 - Índices MSS da amostra}

\begin{tabular}{|c|c|c|c|c|c|c|c|}
\hline & \multicolumn{2}{|c|}{ MÉDIAS } & \multicolumn{3}{c|}{ MÉDIAS } \\
\hline & $\begin{array}{c}\text { QUALIDADE } \\
\text { DESEIADA }\end{array}$ & $\begin{array}{c}\text { NIIVEL } \\
\text { PERCEBIDO }\end{array}$ & MSS & VARIÁVEL & $\begin{array}{c}\text { QUALIDADE } \\
\text { DESEADA }\end{array}$ & $\begin{array}{c}\text { NIVEL } \\
\text { PERCEBID0 }\end{array}$ & MSS \\
\hline Tang1 & 7,76 & 7,477 & $-0,283$ & Prest12 & 8,343 & 7,863 & $-0,48$ \\
\hline Tang2 & 7,879 & 7,524 & $-0,355$ & Prest13 & 8,032 & 7,452 & $-0,58$ \\
\hline Tang3 & 8,106 & 7,775 & $-0,331$ & Seg14 & 8,306 & 7,824 & $-0,481$ \\
\hline Tang4 & 7,92 & 7,481 & $-0,439$ & Seg15 & 8,289 & 7,845 & $-0,444$ \\
\hline Conf5 & 8,029 & 7,64 & $-0,389$ & Seg16 & 8,339 & 8,069 & $-0,269$ \\
\hline Conf6 & 8,415 & 8,044 & $-0,37$ & Seg17 & 8,255 & 7,839 & $-0,416$ \\
\hline Conf7 & 8,111 & 7,795 & $-0,316$ & Emp18 & 8,236 & 7,824 & $-0,412$ \\
\hline Conf8 & 8,259 & 8,031 & $-0,228$ & Emp19 & 8,301 & 8,191 & $-0,11$ \\
\hline Conf9 & 8,071 & 7,82 & $-0,251$ & Emp20 & 8,1 & 7,593 & $-0,507$ \\
\hline Prest10 & 8,217 & 7,912 & $-0,306$ & Emp21 & 8,212 & 7,815 & $-0,396$ \\
\hline Prest11 & 8,232 & 7,673 & $-0,559$ & Emp22 & 8,176 & 7,834 & $-0,342$ \\
\hline
\end{tabular}


sentar coeficiente menor que 0,08 para uma adequação razoável ou menor que 0,05 para uma boa adequação (BROWNE e CUDECK, 1993). Na segunda etapa, foi analisado o modelo estrutural, ou seja, as relações entre os construtos latentes.

\section{RESULTADOS DA PESQUISA}

Nesta seção serão expostos os resultados obtidos na presente pesquisa.

\section{Perfil da amostra}

A amostra inicial decorreu do preenchimento de 557 questionários. Todavia, a amostra final válida considerada neste estudo foi de 351 questionários completamente preenchidos, visto que 57 foram desprezados por não terem sido finalizados e outros 149 , por conterem campos em branco. Note-se que desses 149 formulários inválidos, 27 $(18,1 \%)$ apresentaram valores faltantes na escala RSQ, 91 (61\%) apresentaram valores faltantes na escala Servqual e $31(20,8 \%)$ tinham valores faltantes nas duas escalas.
Dos 351 respondentes retidos na amostra, 214 (61\%) eram do sexo masculino, 278 (79\%) tinham idade variando entre 21 e 50 anos, 302 (86\%) dos respondentes possuíam ao menos o ensino médio completo e $85 \%$ (300 respondentes) recebiam salário acima de $\mathrm{R} \$ 1.000,00$, sendo que a maioria $(80 \%)$ pertencia às classes sociais $\mathrm{A}$ e B, de acordo com o critério Brasil.

\section{Análise fatorial exploratória}

\section{Escala Servqual}

Inicialmente, foram calculadas as Medidas de Superioridade de Serviço (MSS) (PARASURAMAN, ZEITHAML e BERRY, 1994) relativas às variáveis da escala Servqual para que fosse possível realizar as análises fatoriais propostas. Para o calculo dos índices MSS (Tabela 1) seguiu-se a metodologia proposta pelos autores da Servqual, ou seja, calculouse a diferença entre a qualidade percebida e a qualidade desejada de cada item da escala.

Os resultados da AFE sugeriram a eliminação de três variáveis, sendo duas (Conf6 e Emp19) por baixa comunalidade $(<0,4)$ e uma (variável Conf19) por carga fato-

\section{Tabela 2 - AFE escala Servqual}

\begin{tabular}{|c|c|c|c|c|}
\hline VARIÁVEL & PRESTEZA+SEGURANÇA+EMPATIA & ASPECTOS TANGIVEIS & CONFIABILIDADE & \\
\hline Tang1 & & 0,728 & \\
\hline Tang2 & & 0,879 & \\
\hline Tang3 & & 0,472 & \\
\hline Tang4 & & 0,716 & \\
\hline Conf5 & & & $-0,770$ \\
\hline Conf7 & & & $-0,692$ \\
\hline Conf8 & & & $-0,747$ \\
\hline Prest10 & 0,776 & $-0,681$ \\
\hline Prest11 & 0,870 & \\
\hline Prest12 & 0,834 & & \\
\hline Prest13 & 0,855 & & \\
\hline Seg14 & 0,833 & & \\
\hline Seg 15 & 0,719 & & \\
\hline Seg 16 & 0,744 & & \\
\hline Seg 17 & 0,830 & & \\
\hline Emp18 & 0,712 & & \\
\hline Emp20 & 0,838 & \\
\hline Emp21 & 0,711 & & \\
\hline Emp22 & & & \\
\hline
\end{tabular}


rial cruzada em dois fatores. O teste de esfericidade de Barlett foi significativo ao nível de $0,1 \%\left(\chi^{2}=4576,85\right.$; $171 \mathrm{gl}, \mathrm{p}<0,001)$ e o valor do teste KMO foi igual a 0,937. A consistência interna das dimensões foi comprovada por meio de coeficientes alfa de Cronbach que variaram entre 0,793 e 0,948 . Todavia, ao contrário do previsto, foram formados apenas três fatores com autovalores superiores a 1, responsáveis por $66,7 \%$ da variância total, com cargas fatoriais superiores a 0,47 e cargas fatoriais cruzadas inferiores a 0,30. A composição dos grupos fatoriais pode ser analisada na Tabela 2.

No primeiro grupo fatorial ficaram agrupadas todas as quatro variáveis válidas da dimensão Aspectos Tangíveis, no segundo ficaram agrupadas as três variáveis válidas da dimensão Confiabilidade e uma variável da dimensão Presteza e o terceiro grupo reuniu todas as outras onze variáveis - quatro da dimensão Presteza, quatro da dimensão Segurança e três da dimensão Empatia.

\section{Escala RSQ}

A baixa comunalidade $(<0,4)$ e a elevada carga fatorial cruzada implicaram o descarte de quatro variáveis (P10Conf, P28Pol, P11Conf, P23Prob). Entretanto, ao contrário do que foi verificado na Servqual e consistentemente com o que havia sido previsto, a AFE da escala RSQ apontou a existência de cinco fatores com autovalores superiores a 1 , responsáveis por $64 \%$ da variância total, com cargas fatoriais superiores a 0,42 e cargas fa-

Tabela 3 - AFE escala RSQ

\begin{tabular}{|c|c|c|c|c|c|c|}
\hline VARIÁVEL & $\begin{array}{l}\text { INTERAÇÕES } \\
\text { PESSOAIS }\end{array}$ & $\begin{array}{l}\text { ASPECTOS } \\
\text { FÍsICOS }\end{array}$ & $\begin{array}{l}\text { POLITICAS } \\
\text { INTERNAS }\end{array}$ & CONFIANÇA & $\begin{array}{l}\text { SOLUÇÃO DE } \\
\text { PROBLEMAS }\end{array}$ & CRONBACH \\
\hline P1Afis & & 0,762 & & & & \multirow{6}{*}{0,845} \\
\hline P2Afis & & 0,813 & & & & \\
\hline P3Afis & & 0,619 & & & & \\
\hline P4Afis & & 0,443 & & & & \\
\hline P5Afis & & 0,765 & & & & \\
\hline P6Afis & & 0,721 & & & & \\
\hline P7Conf & & & & $-0,778$ & & \multirow{3}{*}{0,836} \\
\hline P8Conf & & & & $-0,787$ & & \\
\hline P9Conf & & & & $-0,671$ & & \\
\hline P12Pess & 0,662 & & & & & \multirow{8}{*}{0,900} \\
\hline P13Pess & 0,777 & & & & & \\
\hline P14Pess & 0,421 & & & & & \\
\hline P15Pess & 0,795 & & & & & \\
\hline P16Pess & 0,652 & & & & & \\
\hline P17Pess & 0,833 & & & & & \\
\hline P18Pess & 0,771 & & & & & \\
\hline P19Pess & 0,708 & & & & & \\
\hline P20Pess & & & & & $-0,661$ & \multirow{3}{*}{0,771} \\
\hline P21Prob & & & & & $-0,745$ & \\
\hline P22Prob & & & & & $-0,695$ & \\
\hline P24Pol & & & 0,502 & & & \multirow{4}{*}{0,701} \\
\hline P25Pol & & & 0,684 & & & \\
\hline P26Pol & & & 0,711 & & & \\
\hline P27Pol & & & 0,790 & & & \\
\hline
\end{tabular}


toriais cruzadas inferiores a 0,30 . O teste de esfericidade de Barlett foi significativo ao nível de $0,1 \%\left(\chi^{2}=4336,41\right.$; $276 \mathrm{gl}, \mathrm{p}<0,001)$ e o resultado do teste KMO foi igual a 0,913 . A confiabilidade das dimensões ficou comprovada por meio de índices alfa de Cronbach que variaram entre
0,701 e 0,900. Como pode ser visto na Tabela 3, a única diferença entre o que foi constatado pela AFE e a escala original foi a variável P20 - originalmente, este item pertence à dimensão de Interações Pessoais, porém, nesta aplicação, ficou agrupada no fator Solução de Problemas.

Tabela 4 - Resultados da validação discriminante com modelos forçados

\begin{tabular}{|c|c|c|c|c|c|c|c|}
\hline MATRIZ FORÇADA & $\chi^{2}$ & gl & RMSEA & CFI & NFI & GFI & RMRpadr \\
\hline- & 507,23 & 145 & 0,08 & 0,92 & 0,89 & 0,87 & 0,05 \\
\hline$\phi(1,2)$ & 655,74 & 149 & 0,10 & 0,89 & 0,86 & 0,83 & 0,09 \\
\hline$\phi(2,3)$ & 661,85 & 149 & 0,09 & 0,89 & 0,86 & 0,84 & 0,08 \\
\hline
\end{tabular}

Figura 1 - Modelo de mensuração escala Servqual

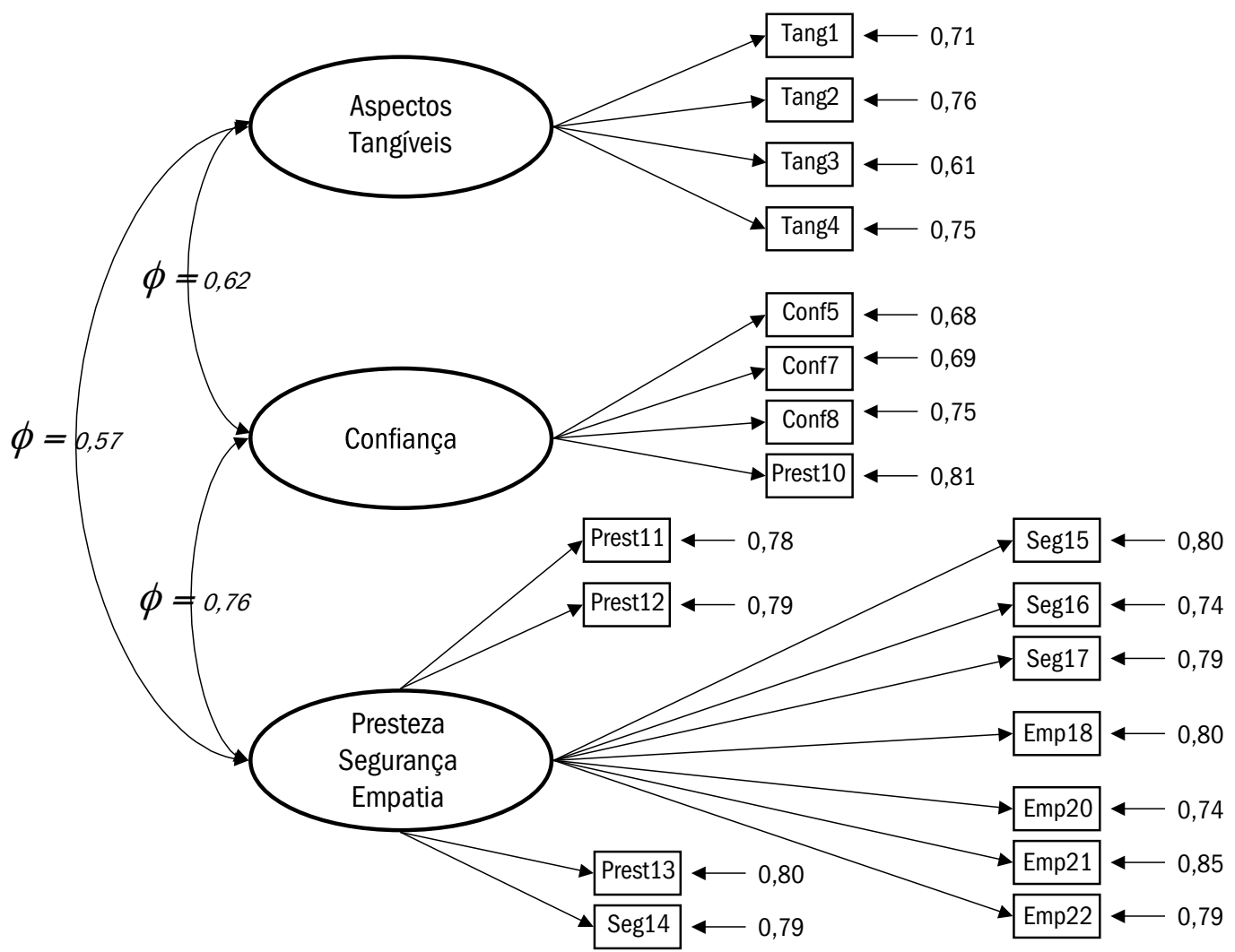

\begin{tabular}{|c|c|c|c|c|c|c|c|c|c|}
\hline \multicolumn{10}{|c|}{ Coeficientes padronizados $(\lambda)$} \\
\hline Tang1 & 0,71 & Conf5 & 0,68 & Prest11 & 0,78 & Seg14 & 0,79 & Emp18 & 0,80 \\
\hline Tang2 & 0,76 & Conf7 & 0,69 & Prest12 & 0,79 & Seg15 & 0,80 & Emp20 & 0,74 \\
\hline Tang3 & 0,61 & Conf8 & 0,75 & Prest13 & 0,80 & Seg16 & 0,75 & Emp21 & 0,85 \\
\hline Tang4 & 0,75 & Prest10 & 0,81 & & & Seg17 & 0,79 & Emp22 & 0,79 \\
\hline
\end{tabular}


Variável dependente - Lealdade

Como se esperava, os três indicadores da variável dependente ficaram agrupados em apenas um fator, com cargas superiores a 0,7, coeficiente alfa de Cronbach de 0,858, teste de esfericidade de Barlett significativo ao nível de $0,1 \%\left(\chi^{2}=497,81 ; 3 \mathrm{gl}, \mathrm{p}<0,001\right)$ e teste $\mathrm{KMO}=0,718$, confirmando a unidimensionalidade dos indicadores desta variável.

\section{Análise fatorial confirmatória}

\section{Modelo de mensuração da escala Servqual}

A Figura 1 representa o modelo de mensuração da escala Servqual gerado a partir da AFC. Os indicadores de ajustamento ficaram dentro de limites aceitáveis $\left(\chi^{2}=507,23 \mathrm{p}<=0,001,145 \mathrm{gl}\right.$, RMSEA = 0,08; CFI $=0,92 ; \mathrm{GFI}=0,87 ; \mathrm{NFI}=0,89 ; \mathrm{RMR}$ padronizado $=$ $0,05)$ e a validade convergente observada foi aceitável, visto que todos os coeficientes lambda ficaram acima de 0,60. O mesmo não ocorreu com a validade discriminante, pois as correlações entre as dimensões Aspectos Tangíveis e Confiança $(\phi 1,2)$, e tam- bém entre Confiança e Presteza+Segurança+Empatia $(\phi 2,3)$, ficaram acima do limite de 0,6 $(\phi 1,2=0,62$ e $\phi 2,3=0,76)$.

Diante desse resultado, realizou-se novo teste de validade discriminante utilizando o método de comparação com modelo forçado e teste de qui-quadrado. Esse método consiste em fixar a correlação entre um par de subconstrutos (Aspectos Físicos e Solução de Problemas, por exemplo) e realizar nova análise do modelo de mensuração ajustado (MCKNIGHT, CHOUDHURY e KACMAR, 2002). Realizase o teste de diferença de qui-quadrados (ANDERSON e GERBING, 1988) para comparar o resultado dos modelos original e forçado. A validade discriminante é determinada se a diferença entre os qui-quadrados for significativa $e$ se ocorrer deterioração dos indicadores de ajustamento, sustentando o modelo original. A Tabela 4 demonstra os resultados obtidos.

A comparação dos qui-quadrados dos modelos forçados frente ao modelo original e os índices de ajustamento obtidos, que foram consistentemente menores, suportaram a validade discriminante do modelo originalmente proposto.

Figura 2 - Modelo de mensuração escala RSQ

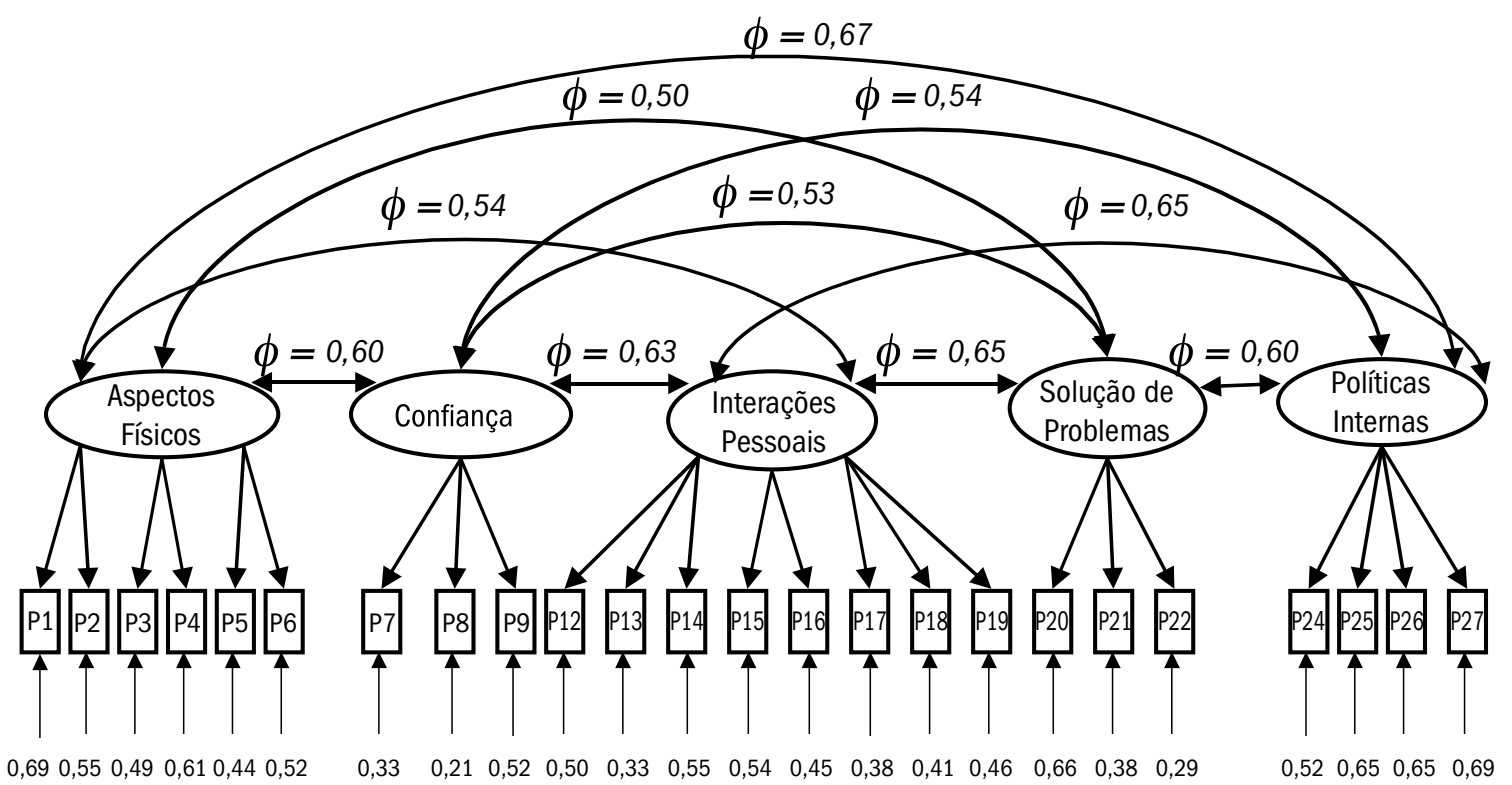

\begin{tabular}{|l|l|l|l|l|l|l|l|l|l|l|l|}
\hline \multicolumn{10}{|c|}{ Coeficientes padronizados $(\lambda)$} \\
\hline P1 & 0,55 & P5 & 0,75 & P12 & 0,71 & P16 & 0,74 & P19 & 0,73 & P24 & 0,69 \\
\hline P2 & 0,67 & P6 & 0,70 & P13 & 0,82 & P17 & 0,79 & P20 & 0,59 & P25 & 0,59 \\
\hline P3 & 0,71 & P7 & 0,82 & P14 & 0,67 & P18 & 0,77 & P21 & 0,79 & P26 & 0,59 \\
\hline P4 & 0,63 & P9 & 0,89 & P15 & 0,68 & & & P22 & 0,84 & P27 & 0,55 \\
\hline
\end{tabular}




\section{Modelo de mensuração da escala RSQ}

Este segundo modelo de mensuração também apresentou indicadores de ajustamento aceitáveis $\left(\chi^{2}=613,18 p\right.$ $<0,001,240 \mathrm{gl}$, RMSEA $=0,06 ; \mathrm{CFI}=0,91 ; \mathrm{GFI}=0,88$; NFI $=0,86 ;$ RMR padronizado $=0,05)$ e cinco das 24 variáveis utilizadas apresentaram coeficientes lambda inferiores a 0,60 , demonstrando que um refinamento mais apurado poderia ser aplicado. Todavia, visando não descaracterizar a escala, optou-se por manter as variáveis apesar desse resultado. Da mesma forma, a validade convergente foi parcial, visto que quatro correlações das dimensões da qualidade do modelo proposto por Dabholkar, Thorpe e Rentz (1996) ficaram acima dos índices recomendados. As correlações entre Confiança e Interações Pessoais $(\phi 3,2)$, Interações Pessoais e Solução de Problemas $(\phi 4,3)$, Interações Pessoais e Políticas Internas $(\phi 5,3)$ e Aspectos Físicos e Políticas Internas $(\phi 5,1)$ apresentaram coeficientes phi entre 0,63 e 0,65 .

Diante disso, o método de comparação com modelo forçado foi novamente utilizado. Os resultados estão disponíveis na Tabela 5.

Também neste segundo teste, os qui-quadrados maiores e a deterioração dos índices de ajustamento permitiram validar o modelo original.

\section{Modelos estruturais}

Diante das diversas relações entre os construtos latentes, vislumbra-se na modelagem de equações estruturais uma técnica bastante adequada para testar os dois modelos de mensuração da QPS. Como demonstrado na Tabela 6, os dois modelos proporcionam boa adequação, embora fique evidente a superioridade dos índices de ajustamento da escala RSQ. A escala desenvolvida por Dabholkar, Thorpe e Rentz (1996) se saiu melhor que a proposta por Parasuraman, Zeithaml e Berry (1985) também para as medidas de ajustamento que levam em conta a parcimônia do modelo (Parsimony Normed Fit Index - PNFI e Parimony Goodness of Fit Index - PGFI).

Observando ainda a Tabela 6 , fica evidente a maior ca-

Tabela 5 - Resultados da validação discriminante com modelos forçados

\begin{tabular}{|c|c|c|c|c|c|c|c|}
\hline MATRIZ FORÇADA & $\chi^{2}$ & gl & RMSEA & CFI & NFI & GFI & RMRpadr \\
\hline- & 603,18 & 240 & 0,06 & 0,91 & 0,86 & 0,88 & 0,05 \\
\hline$\phi(3,2)$ & 733,21 & 241 & 0,06 & 0,88 & 0,84 & 0,87 & 0,15 \\
\hline$\phi(4,3)$ & 735,65 & 241 & 0,07 & 0,88 & 0,86 & 0,86 & 0,14 \\
\hline$\phi(5,3)$ & 684,36 & 241 & 0,06 & 0,89 & 0,87 & 0,87 & 0,13 \\
\hline$\phi(5,1)$ & 700,20 & 241 & 0,07 & 0,89 & 0,86 & 0,86 & 0,12 \\
\hline
\end{tabular}

Tabela 6 - Comparação das medidas de ajuste das escalas

\begin{tabular}{|c|c|c|}
\hline MEDIDAS DE AUSTE & ESCALA SERVQUA & ESCALA RSQ \\
\hline$\chi^{2}$ & $609,80(199 \mathrm{gl})(p<0,00)$ & $731,50(307 \mathrm{gl})(\mathrm{p}<0,00)$ \\
\hline$\chi^{2} / \mathrm{gl}$ & 3,064 & 2,382 \\
\hline RMSEA & 0,07 & 0,06 \\
\hline CFI & 0,90 & 0,91 \\
\hline GFI & 0,86 & 0,87 \\
\hline NFI & 0,86 & 0,86 \\
\hline RMR padronizado & 0,06 & 0,06 \\
\hline PNFI & 0,72 & 0,75 \\
\hline PGFI & 0,68 & 0,71 \\
\hline$r^{2}$ & 0,11 & 0,43 \\
\hline
\end{tabular}


pacidade de explicação da influência da QPS na Lealdade quando utilizada a escala RSQ. A escala desenvolvida por Parasuraman, Zeithaml e Berry (1985) explica apenas 11\% da variação da Lealdade da amostra, enquanto a escala de Dabholkar, Thorpe e Rentz (1996) explica 43\%.

Embora a escala RSQ proporcione melhor explicação da variável dependente do que a escala Servqual, é importante ressaltar que o efeito das dimensões da QPS sobre a Lealdade não foi o mesmo, como demonstram os coeficientes padronizados dos modelos estruturais apresentados na Tabela 7.

As dimensões Aspectos Tangíveis e Confiabilidade da escala Servqual não foram significativas para o modelo. O mesmo ocorreu com as dimensões Aspectos Físicos e Confiança da escala RSQ. Para a escala Servqual, a única dimensão da QPS significativa para o modelo foi o agrupamento de Segurança, Empatia e Presteza, enquanto as dimensões Interações Pessoais, Solução de Problemas e Políticas Internas foram significativas para explicar a variância da Lealdade de amostra. Assim, tomados em conjunto, os resultados sugerem que a escala RSQ é superior à escala Servqual para explicar a variável dependente.

\section{DISCUSSÃO DOS RESULTADOS}

Ambas as escalas confirmaram a multidimensionalidade da QPS. A aplicação da escala Servqual, em contraposição à estrutura original, apontou apenas para três dimensões. Esse fenômeno também havia sido identificado em outros trabalhos acadêmicos, tais como Llosa, Chandon e Orsingher (1998), Veiga e outros (1999), Matos e Veiga (2000) e Rocha e Oliveira (2003). Ficou evidente neste trabalho que a adaptação necessária para que a escala Servqual possa ser utilizada em qualquer natureza de serviços transcende a simples adequação de enunciado dos itens. Seriam necessários vários ajustamentos para conferir à escala maior validade e confiabilidade.

Outro fato confirmado nesta pesquisa é a característica vetorial da qualidade requerida. Assim como identificado por Teas (1994), a tendência à exigência infinita ficou latente na apuração das MSS, que foram todas negativas. Isto é, por maior que seja a qualidade percebida pelos consumidores, muitas vezes estes afirmam que o fornecedor precisa melhorar.

Por outro lado, foi possível identificar as cinco dimensões da qualidade propostas por Dabholkar, Thorpe e Rentz (1996), embora tenha sido necessário o descarte de alguns itens da escala original. A variável P11Conf mensura a confiança do consumidor em relação à acurácia com que são mantidas as informações transacionais realizadas com o varejista, mas, em geral, home centers são lojas em que grande parte das transações comerciais é registrada nos checkouts, por meio de cupons fiscais que não guardam nenhuma informação do comprador, comprometendo assim a avaliação dos consumidores. Por outro lado, a avaliação de disponibilidade de mercadorias (P10Conf) e de cartão de crédito próprio (P28Pol) deveria ter-se ajustado em suas dimensões, pois a primeira é o cerne dos estabelecimentos varejistas e a segunda é um dos diferenciais da empresa pesquisada frente a seus concorrentes. Evidentemente, a baixa correlação desses dois itens com os demais não significa que eles não sejam relevantes para o consumidor, já que se entende que a concessão de crédito é fundamental para potencializar negócios em uma economia emergente, e a concessão

Tabela 7 - Coeficientes padronizados

\begin{tabular}{|l|c|c|}
\hline \multicolumn{1}{|c|}{ CAMINHO } & COEFICIENTE PADRONIZADO & SIG. \\
\hline Escala Servqual & & n.s. \\
\hline Aspectos Tangíveis => Lealdade & $-0,05$ & n.s. \\
\hline Confiabilidade => Lealdade & $-0,05$ & $\mathrm{p}<0,1 \%$ \\
\hline Presteza + Segurança + Empatia => Lealdade & 0,21 & n.s. \\
\hline Escala RSQ & & n.s. \\
\hline Aspectos Físicos => Lealdade & $-0,05$ & $\mathrm{p}<0,1 \%$ \\
\hline Confiança => Lealdade & 0,06 & $\mathrm{p}<0,1 \%$ \\
\hline Interações Pessoais => Lealdade & 0,31 & $\mathrm{p}<0,1 \%$ \\
\hline Solução de Problemas => Lealdade & 0,17 & \\
\hline Políticas Internas => Lealdade & 0,30 & \\
\hline
\end{tabular}


massificada de cartões de crédito próprio pode potencializar a percepção de qualidade de serviços e aumentar os índices de lealdade.

Da mesma forma, o descarte da variável P23Sprob (Os empregados da loja estão habilitados a solucionar pessoalmente as dúvidas e reclamações) aponta para a baixa correlação desse item com os demais que compõem a dimensão. Apesar da diferença entre o pressuposto teórico e o resultado observado, entende-se que, do ponto de vista gerencial, o item pode indicar como está o preparo da equipe de atendimento do varejista e se o operador necessita melhorar ou intensificar o treinamento dos funcionários das lojas. No caso específico, como não foram apontados problemas com a cordialidade do atendimento prestado, mensurada pelas variáveis P19Pess e P20PPess, o treinamento da equipe de atendimento deverá focar os aspectos técnicos do processo de vendas e do conhecimento dos produtos comercializados. Essa característica da escala RSQ reforça a validade externa, pois foi possível mensurar dois aspectos distintos, porém altamente relevantes, da qualidade percebida no atendimento dos operadores varejistas.

Uma possível explicação para o agrupamento incorreto da variável P20Pess na dimensão de Solução de Problemas pode ser a política de atendimento telefônico adotada pela empresa estudada. Na comunicação com o mercado, em todos os tipos de mídia utilizada, somente os telefones do Serviço de Atendimento aos Clientes da companhia são divulgados. A diretoria da empresa entende que, para assegurar o monitoramento e a padronização no atendimento telefônico, a centralização de todos os contatos no callcenter é a melhor opção, e, desse modo, as lojas não divulgam seus números telefônicos. Sendo assim, os clientes que entram em contato por telefone geralmente possuem algum problema a resolver, quer seja de entrega de materiais, quer seja de qualidade de algum produto adquirido.

Mesmo tendo o resultado da AFC evidenciado índices de ajuste razoáveis para as duas escalas, os indicadores da escala RSQ foram ligeiramente, porém consistentemente, superiores em relação aos resultados verificados com a escala Servqual. Isso já não pode ser dito quanto ao poder de explicação da variável dependente proporcionada pelos instrumentos. As diferenças significativas dos coeficientes de correlação (r2) obtidas pelas escalas, por si sós, validam a utilização da escala RSQ como alternativa metodológica para o estudo da QPS e sua correlação com a lealdade dos consumidores.

Outro ponto favorável à adoção da escala RSQ é a parcimônia do instrumento, verificada pelos índices PNFI e PGFI, e também pelo menor número de desconformi- dades no preenchimento dos questionários da pesquisa.

Como ponto em comum, é importante ressaltar que nem as dimensões da qualidade relativas aos ativos e equipamentos da loja (dimensão Aspectos Tangíveis na Servqual e Aspectos Físicos na RSQ), nem as dimensões que mensuram a assertividade na entrega dos serviços, a pontualidade dos compromissos e a guarda correta de registros de clientes e transações (dimensão Confiabilidade na Servqual e Confiança na RSQ) foram significativas e, portanto, não influenciaram a capacidade preditiva das escalas. Isso não significa que as empresas varejistas possam reduzir sua atenção nessas áreas, pois talvez esse fenômeno tenha ocorrido devido ao fato de os consumidores entenderem essas dimensões como pressupostos básicos para que as empresas atuem nesse segmento ou ainda que esse fenômeno pode estar relacionado às especificidades características desse ramo de atividade.

\section{CONSIDERAÇõES FINAIS}

O objetivo principal deste trabalho foi comparar duas escalas de mensuração da qualidade percebida de serviços no ambiente varejista brasileiro. Utilizando-se a escala Servqual (PARASURAMAN, ZEITHAML e BERRY, 1988), classificada como uma das mais tradicionais para a mensuração da QPS, e a escala RSQ (DABHOLKAR, THORPE e RENTZ, 1996), formulada para aplicações específicas em ambientes varejistas, buscou-se verificar empiricamente os pressupostos de validade e confiabilidade de cada escala, além de se propor uma reflexão sobre as características pragmáticas da utilização de um ou outro instrumento.

Os resultados sugerem que, sob a ótica gerencial, as escalas RSQ e Servqual podem indicar pontos frágeis na gestão de serviços de um varejista visando apoiar a formulação de planos de correção para posterior implementação, melhorando o poder competitivo da organização, visando conquistar a lealdade dos clientes. Contudo, os resultados indicam menor complexidade na aplicação da escala RSQ, o que pode ser interessante para esse setor específico.

Do mesmo modo, os resultados suportam as conclusões de estudos anteriores, que indicam a baixa consistência fatorial da escala Servqual (VEIGA e outros, 1999, MATOS e VEIGA, 2000; ROCHA e OLIVEIRA, 2003). Esse fenômeno não foi identificado na aplicação da escala RSQ, na qual se observam as cinco dimensões teóricas da QPS.

Dentre as limitações que marcam a presente pesquisa, três não podem ficar sem menção. A primeira está relacionada ao perfil da amostra estudada. A aplicação da pesquisa apenas entre consumidores frequentadores de 
home centers traz uma limitação referente à extensão da sua utilidade para outros estabelecimentos que revendam materiais para a construção civil. Existe ainda a limitação referente ao processo de coleta dos dados dos pesquisados, feita por meio de uma amostra não probabilística por conveniência, o que impossibilita a generalização dos resultados. A segunda limitação prende-se ao fato de o estudo haver considerado apenas a percepção de qualidade de clientes de uma única empresa, dela extraindo uma amostra em apenas uma cidade brasileira. Finalmente, a terceira é o corte transversal adotado na coleta dos dados. Este estudo não buscou identificar a evolução da relação da QPS com a lealdade no passar do tempo. Recomendase, portanto, que se realizem novas pesquisas utilizando a escala RSQ em outros formatos varejistas e amostras probabilísticas, possibilitando a confirmação de sua estrutura fatorial e a generalização dos resultados encontrados.

Mesmo diante das limitações citadas, algumas contribuições acadêmicas foram alcançadas com o presente estudo. A primeira foi a aplicação, pela primeira vez no Brasil, da escala desenvolvida por Dabholkar, Thorpe e Rentz (1996). A utilização da escala RSQ como ferramenta de mensuração da QPS mostrou-se promissora, mesmo com as validações convergente e discriminante apontando para a necessidade de maior refinamento das variáveis do modelo. As adequadas medidas de ajuste dos modelos de mensuração e estrutural credenciam a escala RSQ para futuras replicações no varejo nacional, em detrimento de outras mais usuais. Ademais, a escala RSQ mostrou-se de fácil aplicação no ambiente a que se propõe e também habilitada a novas aplicações no cenário nacional.

A segunda contribuição foi a comparação entre as escalas Servqual e RSQ, sendo a primeira tida como a mais tradicional já desenvolvida para a mensuração da QPS e despontando a segunda como uma alternativa robusta e parcimoniosa para o mesmo fim.

A terceira contribuição deste trabalho foi visitar novamente o tema QPS e seus impactos na lealdade dos consumidores. Como o acirramento da concorrência entre os varejistas no Brasil gera a forte disputa da preferência dos consumidores, entende-se que o entendimento da QPS poderá gerar diferencial competitivo para as organizações que a utilizarem como estratégia de negócios. Diante disso, recomenda-se que estudos posteriores dêem sequência a este trabalho, replicando a escala RSQ em outros ambientes varejistas, visando conferir a esse instrumento maior validade externa.

A crescente e rápida automação da gestão comercial nivela a capacidade de oferta de produtos e preços entre os grandes operadores, fazendo com que a qualidade dos serviços ofertados aos clientes se torne cada vez mais um fator decisivo na escolha do varejista. O cenário desse novo ambiente acentua a importância de se utilizarem maneiras cada vez mais eficazes para proporcionar aos administradores feedback acurado a respeito da lealdade dos clientes. Espera-se que o presente trabalho tenha proporcionado uma contribuição para que esse objetivo seja alcançado.

\section{REFERÊNCIAS}

ANDERSON, J. C; GERBING, D. W. Structural equation modeling in practice: a review and recommended two-step approach. Psychological Bulletin, v. 103, n. 5, p. 204-215, 1998

BLOSE, J. E; TANKERSLEY, W. B; FLYNN, L. R. Managing service quality using data envelopment analysis. Quality Management Journal On-line, v. 12, n. 2, University of West Florida, 1999

BROWNE, M. W; CUDECK, R. Alternative ways of assessing model fit In BOLLEN, K. A; LONG, L. S. (Orgs) Testing Structural Equation Model. Newbury Park, California: Sage, 1993

CARMAN, J. M. Consumer perceptions of service quality: an assessment of the Servqual dimensions. Jounal of Retailing, v. 66, n. 1, 1990.

CONGRAM, C; FRIEDMAN, M. The AMA Handbook of Marketing for the Services Industries. New York: American Marketing Association, 1991.

CRONIN, J; TAYLOR, S. Measuring service quality: a reexamination and extension. Journal of Marketing, v. 56, n. 3, 1992.

DABHOLKAR, P. A; THORPE, D. I; RENTZ, J. O. A measure of service quality for retail stores: scale development and validation. Journal of the Academy of Marketing Science, v. 24, n. 3, 1996.

DICK, A. S; BASU, K. Customer loyalty: toward and integrated conceptual framework. Journal of the Academy of Marketing Science, v. 22, p. 99-113, 1994

DOMINGUEZ, S. V. O valor percebido como elemento estratégico para obter a lealdade dos clientes. Caderno Pesquisas em Administração, São Paulo, v. 7, n. 4, out./dez. 2000.

EPPLE, D; ZELENITZ, A. The implications of competition among jurisdictions: does Tiebout need politics? Journal of Political Economy, v. 89, n. 6,1981

FINN, D.W; LAMB, C.W. An evaluation of the Servqual scales in retail setting. Advances in Consumer Research, v. 18, p. 483-490, 1991.

GALE, B.T. Gerenciando o valor do cliente: criando qualidade e serviços que os clientes poder ver. São Paulo: Pioneira, 1996.

GRÖNROOS, C. Marketing gerenciamento e serviços. Rio de Janeiro: Campus, 1995 
GUIRY, M; HUTCHINSON, W; WEITZ, B. A. Consumer's evaluation of retail store service quality and its influence on store choice. Working paper. University of Florida, 1992.

HAIR, J. F. e outros. Análise multivariada de dados. Porto Alegre: Bookman, 2005

HUGHEY, D. e outros. Measuring the qualitty of university computer labs using Servqual: a longitudinal study. The Quality Management Journal, v. 10, n. 3, p. 33-44, 2003.

IBGE. Instituto Brasileiro de Geografia e Estatística. Pesquisa anual do comércio, v. 16, 2004. Disponível em: http://www.ibge.gov.br/pac.htm. Acesso em 04.01.2007.

KAUL, S. Measuring retail service quality: examining applicability of international research perspectives in India. Working paper. Indian Institute of Management, Ahmedabad, India, 2005.

KIM, S; JIN, B. Validating the retail service quality scale for US and Korean customers of discount stores: an exploratory study. Journal of Services Marketing, v. 16, n. 3, p. 223-237, 2002.

KLINE, R. B. Principles and Practice of Structural Equation Modeling. New York: The Guilford Press, 2005

LAKATOS, E. M; MARCONI, M. A. Fundamentos de metodologia científica. São Paulo: Atlas, 1996.

LEVIN, J; FOX, J. A. Estatística para ciências humanas. São Paulo: Person Prentice Hall, 2006.

LLOSA, S; CHANDON, J; ORSINGHER, C. An empirical studey of Servqual's dimensionality. The Service Industry Journal, v. 18, n. 2, 1998.

LOURO, M. J. S. Modelos de avaliação de marca. RAE-revista de administração de empresas, v. 40, n. 2, p. 26-37, 2000.

LOVELOCK, P; WRIGHT, L. Serviços: marketing e gestão. São Paulo: Saraiva, 2001.

MACHADO, M. D; QUEIROZ, T. R; MARTINS, M. F. Mensuração da qualidade de serviço em empresas de fast food. Gestão \& Produção, v. 13, n. 2, p. 261-270, 2006

MAJUMDAR, A. A model for customer loyalty for retail stores inside shopping mall - an Indian perspective. Journal of Services Research, Special Issue, p. 48-64, 2005.

MALHOTRA, N. K. Pesquisa de marketing: uma orientação aplicada. Porto Alegre: Bookman, 2001

MCKNIGHT, D. H; CHOUDHURY, V; KACMAR, C. Developing and validating trust measures for e-commerce: an integrative typology. Information Systems Research, v. 13, n. 2, p. 334-359, 2002.

MCMULLAN, R. A multiple-item scale for measuring customer loyalty development. Journal of Services Marketing, v. 19, 6/7, p. 470-481, 2005.

MORGADO, M. G; GONÇALVES, M. N. Varejo: administração de empresas comerciais. São Paulo: SENAC, 1997.
NEL, D; PITT, L. Service quality in a retail environment: closing the gaps. Jounal of General Management, v. 18, n. 3, 1993.

NUNNALLY, J. C. Psychometric Theory, 2. ed., New York: McGraw, 1978.

OLIVER, R. L. A Whence consumer loyalty? Journal of Marketing, v. 63, p. 33-44, 1999

PARASURAMAN, A; ZEITHAML, V. A; BERRY, L. L. A conceptual model of service quality: its implications for future research. Journal of Marketing 28 , p. $41-50,1985$

PARASURAMAN, A; ZEITHAML, V. A; BERRY, L. L. Servqual: a multipleitem scale for measuring consumer perceptions of service quality. Journal of Retailing, v. 64, p. 12-40, 1988.

PARASURAMAN, A; ZEITHAML, V. A; BERRY, L. L. Alternative scales for measuring service quality: a comparative assessment based on psychometric and diagnostic criteria. Journal of Retailing, v. 70, p. 201-230, 1994.

RAMAYAH, T; LEEN, J. Y. A. What drives relationship quality? A study of two retail clothing stores. In: ASIAN ACADEMY OF MANAGEMENT CONFERENCE, 5, 2003, Malasia. Challenges of Globalized Business: The Asian Perspective. Malaysia: AAMC, 2003.

REICHHELD, F. F. A estratégia da lealdade. Rio de Janeiro: Campus, 1996

REICHHELD, F. F. The microeconomics of customer relationships. MIT Sloan Management Review, winter 2006

REIDENBACH, R; SANDIFER-SMALWOOD, B. Exploring perceptions of hospital operations by a modified Servqual approach. Journal of Health Care Marketing, v. 10, p. 47-55, 1990.

SETH, A; MOMAYA, K; GUPTA, H. M. An exploratory investigation of customer loyalty and retention in cellular mobile communication. Journal of Services Research - Special Issue, p. 174-185, 2005.

SHOSTACK, L. How to Design a Service. Chicago: American Marketing Association, 1981.

SOLOMON, M. R. Consumer Behavior: Buying, Having and Being. New Jersey: Prentice Hall, 1998

TEAS, R. Expectations as comparison standard in measuring service quality: an assessment of a reassessment. Journal of Marketing, v. 58, n. 1, 1994.

THORPE, D. Business performance outcomes of service quality: a conceptual model. Developments in Marketing Science, n. 18, p. 159-164, 1994.

URDAN, A. T. Qualidade de serviço: proposição de um modelo integrativo. 1993. Tese de Doutorado em Administração, Faculdade de Economia, Administração e Contabilidade da Universidade de São Paulo, São Paulo, 1993. 